\title{
Seamless Synthetic Aperture Radar Archive for Interferometry Analysis
}

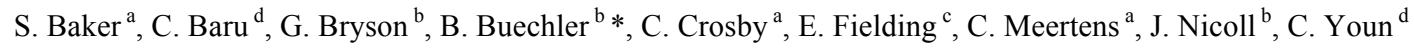 \\ ${ }^{\text {a } U N A V C O, ~ B o u l d e r, ~ C O ~-~(b a k e r, ~ c r o s b y, ~ m e e r t e n s) @ u n a v c o . o r g ~}$ \\ ${ }^{\mathrm{b}}$ Alaska Satellite Facility, Geophysical Institute, University of Alaska Fairbanks, Fairbanks, AK - (gbryson, btbuechler, \\ jbnicoll)@alaska.edu \\ ${ }^{\mathrm{c}}$ Jet Propulsion Laboratory, California Institute of Technology, Pasadena, CA - eric.j.fielding@jpl.nasa.gov \\ ${ }^{\mathrm{d}}$ San Diego Supercomputer Center, University of California San Diego, La Jolla, CA - (baru, cyoun)@sdsc.edu
}

KEY WORDS: Radar, SAR, DEM, Data Mining, Processing, Archiving, Change Detection

\section{ABSTRACT:}

The NASA Advancing Collaborative Connections for Earth System Science (ACCESS) seamless synthetic aperture radar (SAR) archive (SSARA) project is a collaboration between UNAVCO, the Alaska Satellite Facility (ASF), the Jet Propulsion Laboratory (JPL), and OpenTopography at the San Diego Supercomputer Center (SDSC) to design and implement a seamless distributed access system for SAR data and derived interferometric SAR (InSAR) data products. A unified application programming interface (API) has been created to search the SAR archives at ASF and UNAVCO, 30 and 90-m SRTM DEM data available through OpenTopography, and tropospheric data from the NASA OSCAR project at JPL. The federated query service provides users a single access point to search for SAR granules, InSAR pairs, and corresponding DEM and tropospheric data products from the four archives, as well as the ability to search and download pre-processed InSAR products from ASF and UNAVCO.
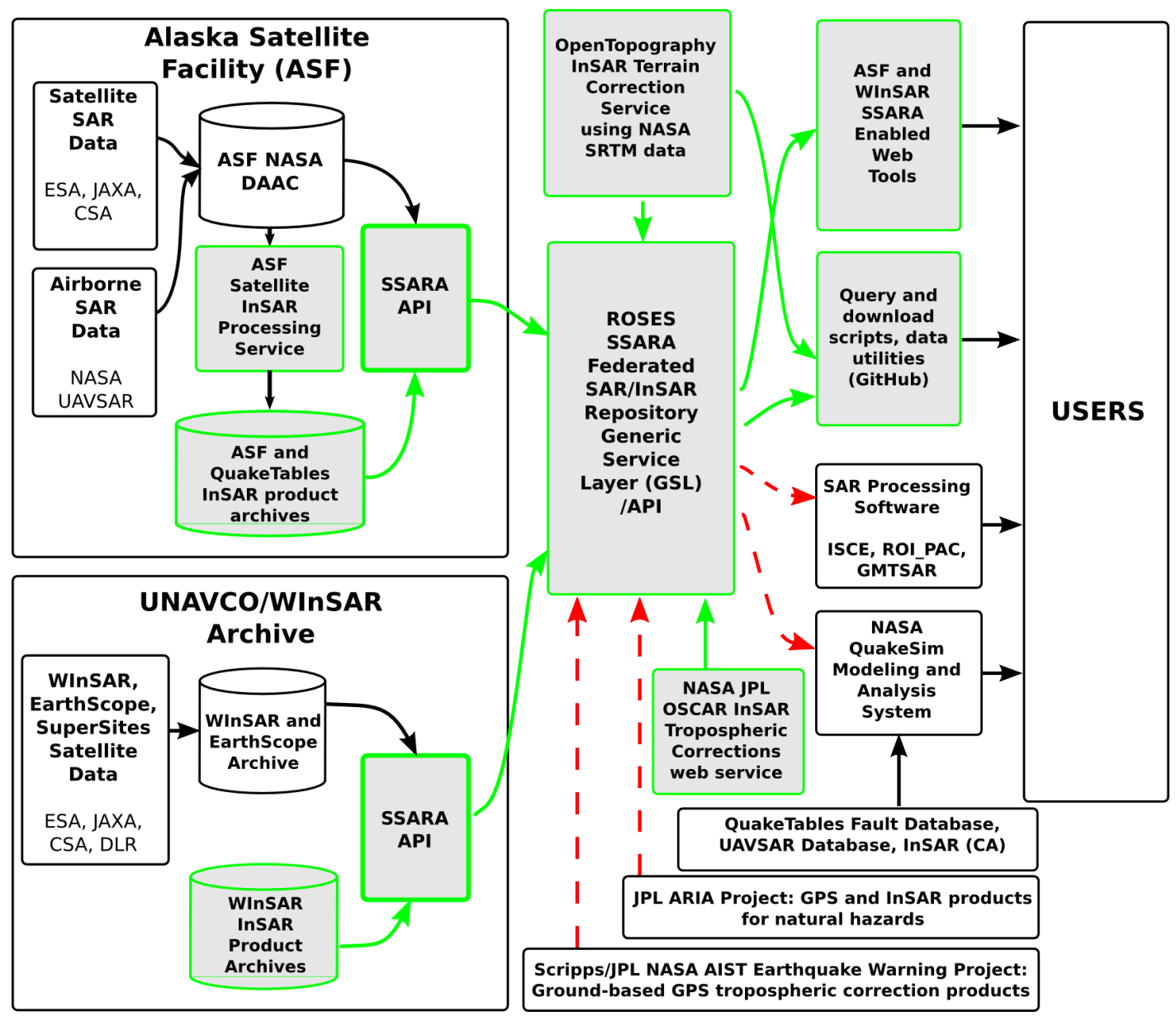

Figure 1. Diagram of SSARA workflow. Grey highlighted boxes show component development as part of the project, green outlines represent tasks already implemented and red outlines represent future implementation.

\footnotetext{
* Corresponding author. This is useful to know for communication with the appropriate person in cases with more than one author.
} 


\section{INTRODUCTION}

\subsection{Overview}

Differential interferometric synthetic aperture radar (InSAR) is used to measure displacements on Earth's surface to a precision of a few centimeters or less. InSAR data are largely used to study deformation caused by earthquakes, volcanic eruptions, glacier movements, landslides, and subsidence. They may also be used to examine the distribution of water vapor in the atmosphere, among other applications.

An interferogram is formed from two or more SAR granules acquired at different times that cover the same area of ground and have the same acquisition geometry. These granules are referred to as a master image and slave image(s). Interferometry from a single pair of SAR images contains direct topographic and atmospheric effects that must be removed in order to create a quality InSAR product.

The SSARA application programming interface (API) enables users to search and download InSAR master and slave granules from the SAR archives at ASF and UNAVCO (http://www.unavco.org/ws/brokered/ssara/sar/search); corresponding DEMs from Open Topography and tropospheric data from JPL

(http://www.unavco.org/ws/brokered/ssara/insar/selectpairs); and sample standardized InSAR data products from ASF and UNAVCO

(http://www.unavco.org/ws/brokered/ssara/insar/search).
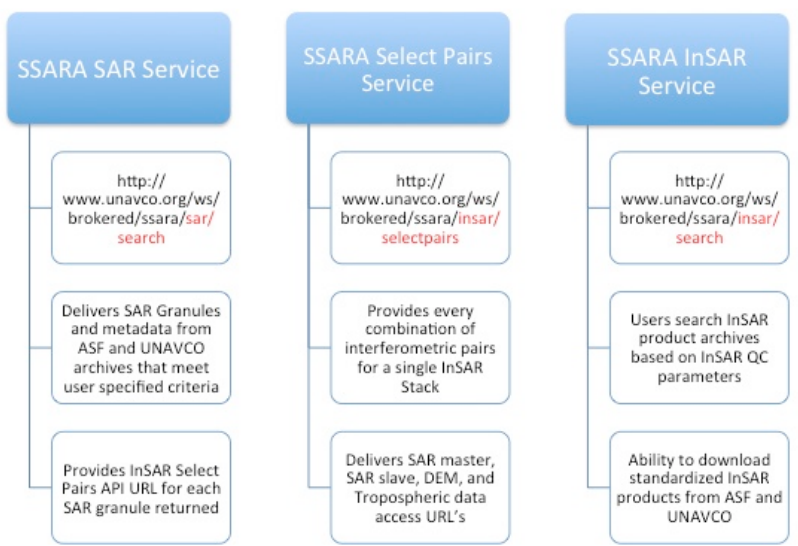

Figure 2. SSARA API Services and corresponding uniform resource locators (URL).

\subsection{Objectives}

The SSARA collaboration set the following objectives:

- Develop and implement a single access point for metadata query and data product download capabilities from distributed airborne and spaceborne SAR archives at ASF and UNAVCO

- Define and implement new quality control (QC) parameters and products to enhance the usability of data from existing NASA-funded data collections

- Develop standard formats for image products such as interferograms, tropospheric measurements, and terrain corrections
- Implement a web services enabled terrain correction service using NASA SRTM data

- Enhance ASF SAR interferometric processing service to access distributed SSARA data collections, utilize terrain correction service, and generate enhanced QC products

- Establish processed InSAR data products and QC product archives at ASF and UNAVCO

\section{DATA ARCHIVES}

\subsection{SAR Data Archive at ASF}

The ASF of the Geophysical Institute (GI) at the University of Alaska Fairbanks, downlinks, archives, and distributes satellitebased data to scientific users around the world. ASF's NASAsponsored Distributed Active Archive Center (DAAC) specializes in SAR data collection, processing, archiving, and distribution. These data are processed on-demand through URSA (https://ursa.asfdaac.alaska.edu/) or can be downloaded as a standardized Level 0, Level 1 Complex, or Level 1 Detected products from the ASF Data Pool via Vertex (https://vertex.daac.asf.alaska.edu/) or the ASF API (http://api.daac.asf.alaska.edu/services/search/param).

The ASF Data Pool contains over 500 TB of online readilydownloadable data. Those datasets useful for interferometry can be searched publicly, including browse products for selected datasets, and Keyhole Markup Language (KML) files of locations for all datasets, but require user authentication to access the data themselves. This authorization is through on-line initiated proposal submission services, coordinated by ASF, with access granted through NASA.

\subsection{SAR Data Archive at UNAVCO}

UNAVCO is a non-profit university-governed consortium that operates the National Earth Science Geodesy Advancing Geosciences and EarthScope (GAGE) Facility.

UNAVCO is home to the Western North America InSAR Consortium (WInSAR) archive (http://winsar.unavco.org) that was originally constructed to handle a limited ERS-1 and ERS-2 satellite data collection. In recent years, the WInSAR archive has expanded to include Envisat data brining the total archive holdings to approximately 32,000 SAR scenes from ESA. More recently, the archive has been expanded to manage TerraSAR-X data for WInSAR PIs with approved DLR proposals to facilitate easier access and data sharing with individual co-investors (CoIs) on the projects. There are over 1200 scenes from 15 PI proposals. UNAVCO also operates the EarthScope SAR archive, which holds over 105,000 frames of ERS-1, ERS-2, Envisat, and Radarsat-1 data. UNAVCO and WInSAR worked with the European Space Agency (ESA) to promote and develop the Group of Earth Observation (GEO) Geohazard Supersites and Natural Laboratories (GSNL) (http://supersites.earthobservations.org) data sharing concept and to prototype infrastructure for SAR data. UNAVCO ordered data from the ESA and access to the data are provided through the Virtual Archive 4 after self-registration. (http://eo-virtualarchive4.esa.int/). UNAVCO also archives COSMO-SkyMed and Radarsat-2 data on behalf of the GSNL for select locations.

All data holding can be searched publicly, but user registration and authentication is required to gain access to SAR data at UNAVCO. To download WInSAR ESA or EarthScope data, 
users must be affiliated with a full or adjunct I consortium member institution and sign the data use agreements from the space agencies. For the TerraSAR-X and GSNL data holdings at UNAVCO, users must contact the PI on the proposal and be added as a Co-I in order to gain access to the data. WInSAR also offers a mechanism for Consortium members to request ordering of archived scenes over North America for ERS and Envisat data that are not yet in WInSAR holdings.

\subsection{DEM Data Archive at OpenTopography}

The NSF-funded OpenTopography facility (OT, http://www.opentopography.org) provides community access to high-resolution (e.g., lidar) and other (e.g., SRTM) Earth science-oriented, topography data, and related tools and resources (Crosby et al., 2011). OT has developed a cyberinfrastructure platform built on a service oriented architecture (SOA) to enable efficient online access to these datasets along with web based processing tools to generate derived products including digital elevation models (DEMs) (Krishnan et al., 2011). All data access functions and processing algorithms are packaged as web services deployed across multiple OpenTopography commodity clusters, leveraging the open source OPAL toolkit (http://sourceforge.net/projects/opaltoolkit/). OPAL is an opensource toolkit developed by the National Biomedical Computation Resource (NBCR) and the San Diego Supercomputer Center (SDSC) that enables users to wrap scientific applications running on cluster and Grid resources as Web services. Since the service-oriented architecture of OT supports a distributed environment and service interoperability with its loose coupling and published APIs using standards based communication protocols, it is beneficial for other communities to be easily integrated with the topography datasets' processing capabilities for the derivation of higherlevel analysis products.

Current OpenTopography LiDAR point cloud data holdings alone exceed 18 terabytes (in compressed binary format) covering approximately $170,476 \mathrm{~km}^{2}$ of important geologic features such as the San Andreas Fault, Yellowstone, Tetons, Yosemite National Parks, etc. Global datasets such as the SRTM 30 and 90 meter datasets are also available. Typically, users interact with the portal interface to perform sub-selections of the datasets and run processing algorithms on their area of interest to produce derivative products like DEMs, hillshade, visualizations, and morphometric products such as slope (Youn et al., 2014).

\subsection{Tropospheric Data Archive at JPL}

SSARA builds upon the web services provided by the JPL Online Services for Correcting Atmosphere in Radar (OSCAR) project (Allmen et al., 2011). OSCAR locates, retrieves, and merges multiple data sets to derive an optimal, best estimate of tropospheric delay (Table 3). The service produces maps of the precipitable water vapor for a single date. However, in order to deliver proper results, data must be acquired during the daytime under cloud-free conditions. When combined with SAR imagery OSCAR provides estimates of radar propagation delay for each SAR scene

One of the largest sources of errors in InSAR measurements are variations in radar wave propagation through the atmosphere. In the troposphere, the propagation of radio waves is affected by the total mass of atmosphere along the path (often called the "dry" delay) and the integrated amount of water vapor along the path (usually called the "wet" delay). The total mass of the atmosphere is easily calculated from the surface pressure. The atmospheric water vapor content can be measured by satellite instruments, such as the MODIS instruments on Terra and Aqua (Li et al., 2005) or estimated from weather models. GPS ground station data processing for precise locations includes estimation of the tropospheric path delay due to both the dry and wet components, which is exactly the same delay that affects InSAR measurements (Li et al., 2006). The Scripps/JPL GPS AIST project is routinely producing these tropospheric delay products for the continuous GPS stations that they process.

\begin{tabular}{|c|c|}
\hline $\begin{array}{l}\text { - Time series filtering or estimation } \\
\text { - Correlation of phase with topography }\end{array}$ & $\begin{array}{l}\text { InSAR } \\
\text { Derived }\end{array}$ \\
\hline $\begin{array}{l}\text { - CGPS (Continuous Global Positioning } \\
\text { System) zenith wet delay interpolated } \\
\text { spatially (and temporally) }\end{array}$ & $\begin{array}{l}\text { Ground- } \\
\text { Based }\end{array}$ \\
\hline $\begin{array}{l}\text { Total column water vapor from } \\
\text { absorption of reflected near IR (MODIS } \\
\text { and MERIS) } \\
\text { - Water vapor measurements (profiling and } \\
\text { total column) from thermal IR and MW } \\
\text { (AIRS, MODIS, AMSU) }\end{array}$ & $\begin{array}{l}\text { Remote- } \\
\text { Sensed }\end{array}$ \\
\hline $\begin{array}{l}\text { - European Center for Medium Range } \\
\text { Weather Forecasting (ECMWF) } \\
\text { - NOAA NCEP North American } \\
\text { Mesoscale Model (NAM) }\end{array}$ & $\begin{array}{l}\text { Numerical } \\
\text { Weather } \\
\text { Forecast } \\
\text { Models }\end{array}$ \\
\hline
\end{tabular}

Table 3. Datasets utilized by OSCAR to derive tropospheric water vapor corrections for InSAR.

\section{INSAR ARCHIVE}

One of the primary products that can be produced with SAR data is an interferogram, a difference of a pair of SAR scenes from repeated satellite or aircraft radar scans that are typically separated from days to years apart. The resulting phase differences between the two SAR images is called Interferometric SAR, or InSAR, and provides an estimate of surface differences at the sub-centimeter level. InSAR from a single pair of SAR images contains direct topographic and atmospheric effects that must be removed in order to create a quality InSAR product.

Currently, the bulk of InSAR processing is done by individual scientists, often using the Repeat Orbit Interferometry Package (ROI_PAC), the InSAR Scientific Computing Environment (ISCE), or the Generic Mapping Tool SAR software (GMTSAR). ROI_PAC and ISCE are NASA-funded software packages develope $\bar{d}$ by JPL/Caltech and distributed at no charge for non-commercial use. UNAVCO distributes the ISCE software for WInSAR member institutions (https://winsar.unavco.org/isce.html). GMTSAR is developed at Scripps Institute of Oceanography and the software is distributed under a GPL license. To help broaden the InSAR user community, UNAVCO supports annual InSAR processing short courses that teach users how to generate interferograms using ISCE and GMTSAR, but proficiency can take years to develop. Among the obstacles that InSAR users must overcome are the variety of tools for accessing data and complex user authorization rules, software maintenance, the need to access digital elevation models (DEM) to perform terrain corrections, and the need to assess effects of varying troposphere conditions 
on an image pair.

The SSARA project reduces these obstacles by providing users with the means to search and download SAR pairs, and corresponding DEM and tropospheric data using a single API (http://www.unavco.org/ws/brokered/ssara/insar/selectpairs). In addition, SSARA created an archive of approximately 700 downloadable sample InSAR products at the ASF and an archive of Principal Investigator contributed interferograms at UNAVCO. Products from the InSAR archives are available to users from the unified SSARA API access point (http://www.unavco.org/ws/brokered/ssara/insar/search).

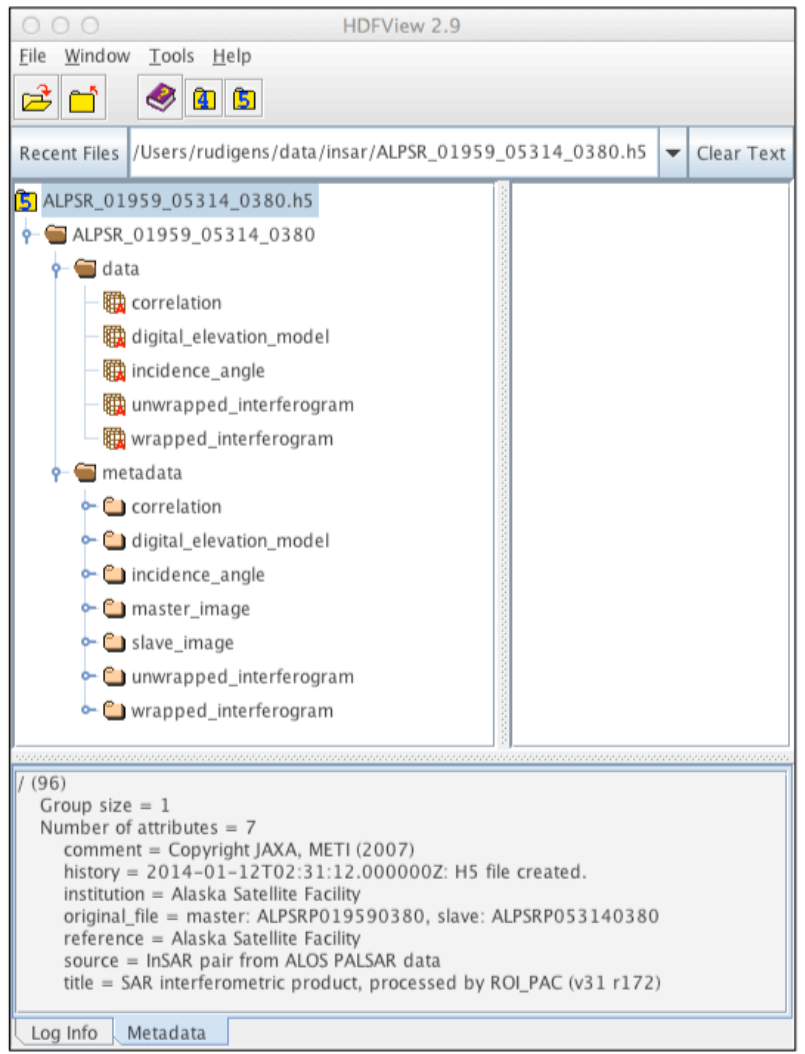

Figure 4. HDF5 Data and Metadata Structure as displayed in HDFView.

\subsection{Product Contents and Format}

Prototyped standardized InSAR products are distributed in HDF5 format and structured to follow the NetCDF Climate and Forecast (CF) Metadata Convention. The sample HDF5 InSAR products contains a data section with imagery and a metadata section with detailed information that corresponds to each data set (Figure 4).

There are five sets of data included with the sample InSAR products: correlation map, wrapped interferogram, unwrapped interferogram, digital elevation model, incidence angle map, and corresponding metadata. The correlation maps vary in value range between 0 , complete loss of correlation, to 1 , perfect correlation between master and slave image (Figure 5). The wrapped interferogram contains phase information with fringes that limit the values within a range of $-\pi$ and $+\pi$. Phase unwrapping produces continuous phase values that can be directly linked to terrain height with the baseline information. A DEM is used to remove topographic effects from the InSAR product and an incidence angle map derived from the DEM is added for advanced analysis purposes. The metadata section contains the metadata for the correlation map, wrapped and unwrapped interferogram, DEM, and incidence angle map, as well as metadata for the original SAR master and slave data used to generate the sample InSAR product.

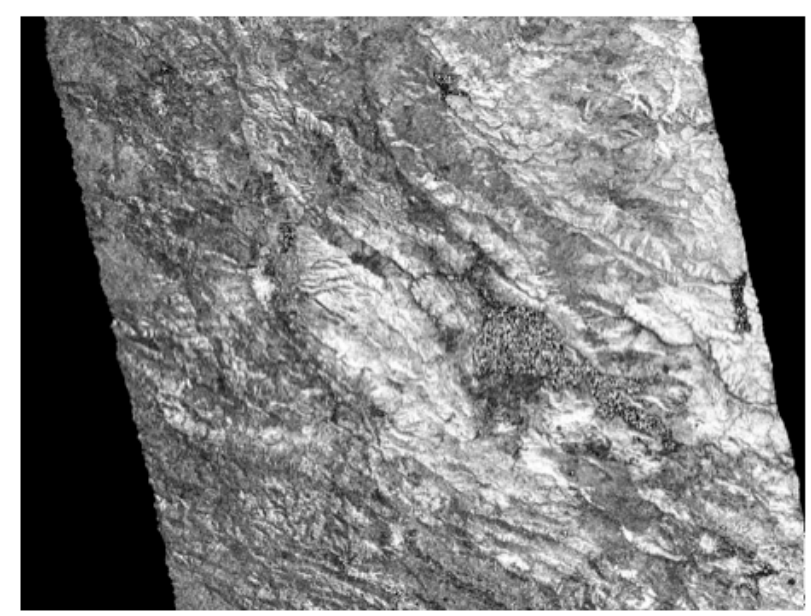

Figure 5. Correlation Map.

In addition to the HDF5 sample InSAR product, ASF provides a browse image of the unwrapped interferogram in JPEG format and four KML overlay files in compressed KMZ format: amplitude, wrapped and unwrapped interferogram, and correlation.

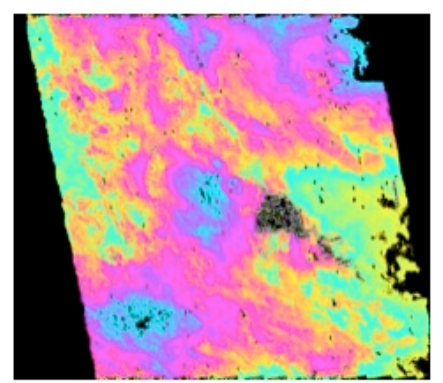

Figure 6. Browse of unwrapped interferogram.

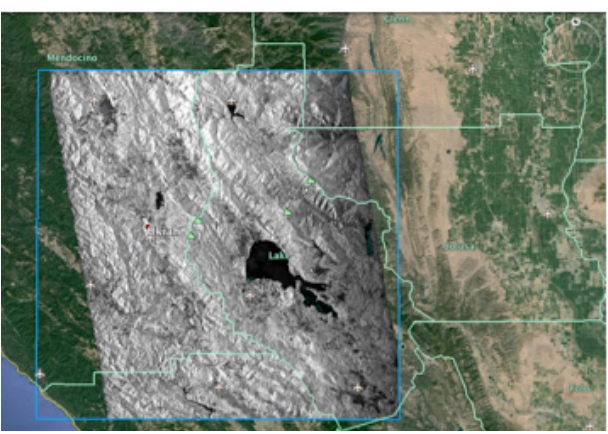

Figure 7. KML overlay of terrain corrected SAR amplitude image.

The naming convention for the sample InSAR products is AAAA_BBBBB_CCCCC_DDDD; where AAAA is the mission identifier, $\mathrm{BBBBB}$ is the orbit number of the SAR master image, CCCCC is the orbit number of the SAR slave image, and DDDD is the frame number of the master and slave images. An example of the files delivered as part of an InSAR product are 
displayed in Table 8 . The sample InSAR products are currently distributed with XML and ISO 19115 compliant metadata (Figure 9)

\begin{tabular}{|l|l|}
\hline HDF5 Product: & ALPSR_06713_08055_0770.h5 \\
\hline Browse Image: & ALPSR_06713_08055_0770.jpg \\
\hline XML Metadata: & ALPSR_06713_08055_0770.xml \\
\hline KML Overlay: & ALPSR_06713_08055_0770.amp.kmz \\
& ALPSR_06713_08055_0770.cor.kmz \\
& ALPSR_06713_08055_0770.int.kmz \\
& ALPSR_06713_08055_0770.unw.kmz \\
\hline Log File: & ALPSR_06713_08055_0770.log.txt \\
\hline
\end{tabular}

Table 8. Example files included in the sample standardized InSAR products.

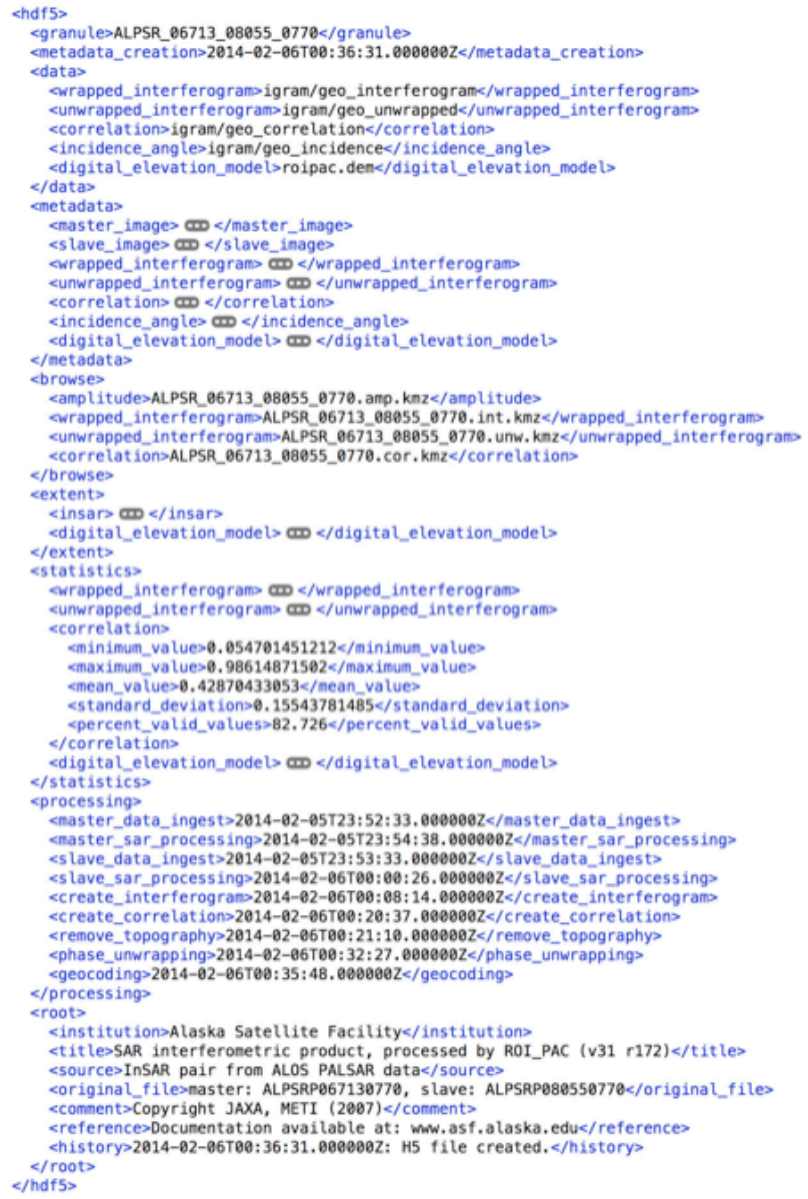

Figure 9. Example of XML metadata file used to generate ISO compliant metadata.

\subsection{Calculating Baselines}

In principle, SAR data from all satellites in the ASF and UNAVCO archives should be suitable for InSAR, as long as the image pair adheres to some basic rules: the data needs to be acquired by the same satellite, in the same beam mode, and with the same look direction. There are a few exceptions to this rule. The tandem mode of the ERS-1 and ERS-2 satellites provides data very suitable for InSAR applications because both satellites meet the criteria above and have a favorable temporal baseline (data acquired one day apart). All the data in the ASF archive, except RAMP data, are right looking. This means that RAMP data cannot be combined with any other Radarsat-1 data, even if those had been acquired with the same beam mode. ScanSAR data, available for R1 or ALOS PALSAR, are another special case, as they are formed by combining several beam modes in one data set.

An InSAR stack is composed of all granules that cover the same geographic region, are from the same platform, and were acquired with the same beam mode. Theoretically, any two granules in an InSAR stack may be used to create an interferogram, as long as the baseline is not beyond a certain critical length.

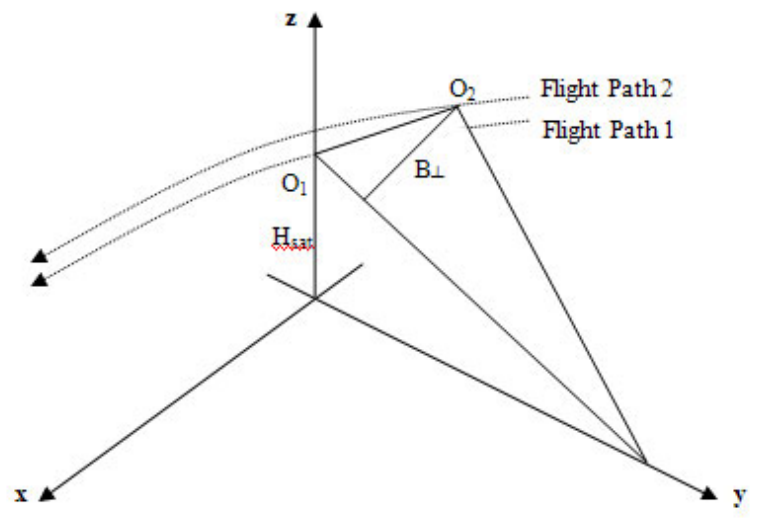

Figure 10. Baseline length is the temporal and spatial distance between two satellite observations

Perpendicular Baseline $\left(B_{\perp}\right)$ is the spatial distance between the first and second observations measured perpendicular to the look direction. It gives an indication of the sensitivity to topographic height, the amount of decorrelation due to phase gradients, and the effectiveness of the phase unwrapping. The longer $B_{\perp}$, the weaker the coherency and the lower the sensitivity to height changes (Hanssen, R.). Temporal Baseline is the time difference between the first and second acquisition. To minimize decorrelation, the temporal baseline should be as short as possible. However, this may not be the case for studies of continuous deformation such as earthquake or volcano monitoring.

Critical baseline is the maximum horizontal separation between two satellite orbits at which the interferometric correlation becomes zero. It is a function of wavelength $(\lambda)$, incidence angle $\left(\theta_{\text {inc }}\right)$, topographic slope $(\zeta)$, range bandwidth $\left(B_{R}\right)$, and range (R):

$$
B_{\perp \text { critcal }}=\lambda\left(B_{R} / c\right) R_{1} \tan \left(\theta_{\text {inc }}-\varsigma\right)
$$

Standard interferometric processing techniques can only be applied when the baseline of the interferometric pair is well below the critical baseline. Advanced interferometric processing techniques, such as persistent scatterer analysis, are capable to overcome this limitation.

\subsection{Best InSAR Pair Selection Algorithm}

Interferometric pairs are selected to minimize both perpendicular baseline and time span. Similar to the work by Pepe and Lanari [2006], Delaunay triangulation is used to 
select interferometric pairs in the perpendicular-temporal baseline space to create an interconnected network of interferograms. The temporal baseline values are scaled by the ratio of perpendicular baseline to temporal baseline to generate an even space for the Delaunay triangulation. Further refinement of the network is done by eliminating pairs that exceed a perpendicular baseline threshold (typically $600 \mathrm{~m}$ for ERS-1/2, Envisat, and Radarsat; $2500 \mathrm{~m}$ for ALOS).

\section{SSARA API}

The SSARA API provides a set of web services to search and retrieve metadata for pertinent SAR holdings using a simple calling convention. Queries are sent as HTTP GET or HTTP POST requests for resources based upon API parameters supplied in the request. Each query requires the base URL (http://www.unavco.org/ws/brokered/ssara/), the resource (sar/search, insar/search, or insar/selectpairs), and a list of query parameters. The API returns the search result in the open, text-based data exchange format JSON that includes metadata for scenes as well as URLs for direct data downloading. The SSARA services can be accessed programmatically using a number of languages (Python, Perl, Java, etc.), common command line utilities such as wget or curl, or any web browser. A list of the query fields is provided in Table 12 as well as on the GitHub wiki page (https://github.com/bakerunavco/SSARA/wiki/SSARA-API).

\subsection{SAR Service}

Using the simple calling convention, the SAR service search URLs are constructed as follows:

http://www.unavco.org/we/brokered/ssara/sar/search?<QUERY FIELD $>=$ value \&...

The response returned in JSON format includes status, count, message, request parameters, and a results array with the SAR scenes. The JSON for each SAR scene has a common set of fields relevant to SSARA as well as archive specific fields necessary for backwards compatibility.

Query URL:

http://www.unavco.org/ws/brokered/ssara/sar/search?platform= ENVISAT\&relativeOrbit $=200 \&$ maxResults $=1$

Query Result:

\{"status": "SUCCESS", "count": 1, "message": "", "resultList": [\{"beamMode": "IM", "flightDirection": "D", "sceneSize": "frame", "sarSceneId": 227711, "finalFrame": 3195, "sceneDate": 1076963004862, "configurationName": "Standard Beam 2", "frequency": 5.331, "thumbnailUrl": "http://facility.unavco.org/data/sar/lts2/lt/thumbnails/ENVISAT $-1 / 10274 / 200 / 3195 / 20040216$ T20231812020040216T202333210 D T-XI0B.jpg", "insarGrouping": 19, "firstFrame": 3195, "collectionName": "WInSAR ESA", "insarStackSize": 6, "platform": "ENVISAT", "absoluteOrbit": 10274, "polarization": "VV", "start": 1076963004862, "formatName": "ENVISAT", "stopTime": "2004-02-16 20:23:39", "missionName": "ENV1", "sensor": "ASAR", "baselinePerp": null, "status": "archived", "catSceneId": null, "doppler": -119.118162, "faradayRotation": null, "track": 200, "stop": 1076963019942, "stringFootprint": "POLYGON (($154.89246120 .393759,-155.09213519 .499568,-156.116017$ $19.709568, \quad-155.919237 \quad 20.603759, \quad-154.892461$ 20.393759))", "sceneId": 227711, "satelliteName": "ENVISAT", "startTime": "2004-02-16 20:23:24", "insarPairs": "http://www.unavco.org/ws/brokered/ssara/insar/selectpairs?pla tform $=$ ENVISAT\& relativeOrbit $=200 \&$ frame $=3195 \&$ beamSwat h=S2", "lookAngle": 20.138, "sceneDateString": "2004-02-16", "incidenceAngle": 22.95, "orbit": 10274, "processingLevel": "L0", "downloadUrl": "https://facility.unavco.org/data/sar/lts1/winsar/ENV2/200/3195 /ASA_IM_0CNPDK20040216_202324_000000152024_0020 0_10274_2388.N1", "processingTypeName": "L0", "beamSwath": "S2", "lookDirection": "R", "relativeOrbit": 200\}], "requestParameters": \{"platform": "ENVISAT", "relativeOrbit": "200", "maxResults": "1"\}\}

\subsection{Select Pairs Service}

The select pairs service uses the same simple calling conventions as the SAR service. Search URLs are constructed as follows:

http://www.unavco.org/we/brokered/ssara/insar/selectpairs? $<\mathrm{Q}$ UERYFIELD $>=$ value \&...

The response returned in JSON format includes status, interferogram count, sar scene count, stack count, message, request parameters, and a results array with the InSAR pairs. The JSON for each InSAR pair includes URLs for master and slave scenes, zenith path delay difference, and DEM for each interferogram. The service currently returns every combination of interferometric pairs for a single InSAR stack, and further development of the service will provide parameters for limiting results based on temporal and perpendicular baselines for interferograms.

Query URL:

http://www.unavco.org/ws/brokered/ssara/insar/selectpairs?platf orm $=$ ENVISAT\&relativeOrbit $=200 \&$ frame $=3195 \&$ beamSwath $=\mathrm{S} 2$

Query Result:

"status": "SUCCESS", "requestParameters": \{"platform": "ENVISAT", "frame": "3195", "relativeOrbit": "200", "beamSwath": "S2"\}, "ifgramCount": 807, "sarCount": 51, "stackTotal": 1, "message": "', "insarPairs": [\{"slave": "https://eo-virtual-

archive4.esa.int/supersites/ASA_IM_0CNPDE20070205_2023 03_000000692055_00200_25805_0136.N1", "platform": "ENEISAT", "master": "http://eo-virtualarchive2.esa.int/Hawaii/ASA_IM_0CNPDE20070205_202303 $00000069205500200258050136 . N 1 ", \quad$ "dem": "http://otdata1.sdsc.edu:9090/otr/getdem?demtype=SRTM90\&north=22. 051017 \& south $=16.905038$ \& east $=-154.506737 \&$ west $=$ -

156.818975", "tropo":

"http://oscar.jpl.nasa.gov/service/zpddm?\&lat1=22.051017\&lat $0=16.905038 \&$ lon $1=-154.506737 \& \operatorname{lon} 0=-$

156.818975\& $\mathrm{t} 0=2010-09-27 \mathrm{~T} 20: 23: 09 \& \mathrm{t} 1=2010-07-$

19T20:23:12", "relativeOrbit": 200, "firstFrame": 3177$\}, \ldots\}$

\subsection{InSAR Service}

All ASF standardized sample ALOS PALSAR InSAR products have been processed with a customized version of ROI_PAC (version 3.1, revision 172). The SAR interferometric products have been terrain corrected using a digital elevation model provided by Open Topography. In the future, tropospheric effects will be corrected using water vapor model outputs based on MODIS data provided by the Online Services for Correcting Atmosphere in Radar (OSCAR) of the Jet Propulsion Laboratory (JPL). 


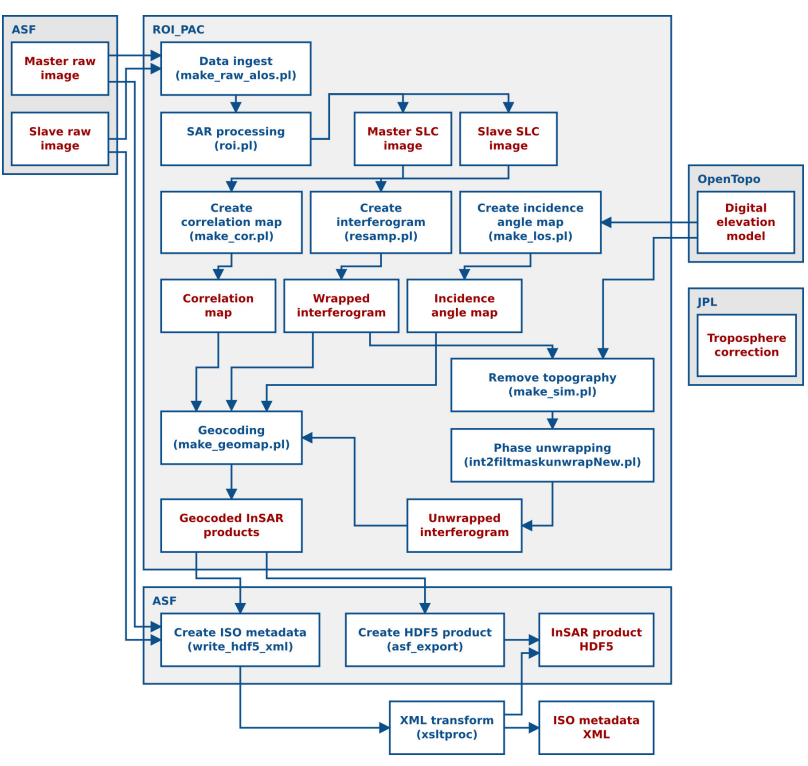

Figure 11. InSAR Processing Flow Diagram

(https://www.asf.alaska.edu/sar-data/insar/product-guide/).

Sample standardized InSAR data products may be searched and downloaded from ASF and UNAVCO SAR archives using URLs in the form:

http://www.unavco.org/ws/brokered/ssara/insar/search? $<$ QUER YFIELD $>=$ value \&...

The response returned in JSON format includes status, count, request parameters, and a results array with the interferograms.

Query

URL:

http://www.unavco.org/ws/brokered/ssara/insar/search?platfor $\mathrm{m}=\mathrm{ALOS} \&$ relativeOrbit $=291 \&$ frame $=370 \&$ beamSwath $=7$

Query

Result:

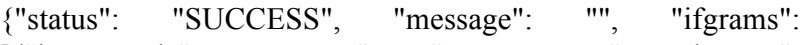
[\{"beamMode": "FBD", "granuleType": "ALOS_PALSAR_INSAR", "frameNumber": "370", "flightDirection": "ASCENDING", "sceneSize": null, "sarSceneId": null, "finalFrame": "370", "sceneDate": "201006-28 08:58:54", "nearStartLat": 18.989, "configurationName": "ALOS PALSAR sensor: High Resolution Observation Mode (dual polarization)", "frequency": null, "farEndLon": -155.089, "centerLat": 19.2973, "processingDescription": "Terrain Correct HDF5 InSAR Product", "sceneDateString": null, "percentTroposphere": "0", "farStartLat": 19.1, "insarGrouping": "1496947", "firstFrame": "370", "thumbnailUrl": "none", "nearEndLon": -155.705, "processingType": "L1", "absoluteOrbit": "NA", "masterGranule": "ALPSRP229060370", "nearStartLon": 155.602, "varianceTroposphere": null, "polarization": "HH+HV", "platform": "ALOS", "formatName": null, "stopTime": "2010-06-28 08:58:54", "missionName": "ASFA3-FBD", "farStartLon": -154.988, "nearEndLat": 19.494, "offNadirAngle": "34.3", "sensor": "SAR", "baselinePerp": "113.306257362648679492",

"slaveGranule": "ALPSRP235770370", "status": null, "catSceneId": null, "doppler": "NA", "fileName": "ALPSR_22906_23577_0370.h5", "beamModeType": "FBD", "faradayRotation": - "NA", "track": null, "processingTypeDisplay": "(BETA) HDF5 InSAR Product", "collectionName": "ASF-A3-FBD", "flightLine": "291",
"stringFootprint": "POLYGON ((-155.602 18.989, -154.988 19.1, -155.089 19.605, -155.705 19.494, -155.602 18.989))", "sceneId": "ALPSR_22906_23577_0370", "percentCoherence": "74.971", "granuleName": "ALPSR_22906_23577_0370", "startTime": "2010-05-13 08:59:13", "productName": "ALPSR_22906_23577_0370", "percentUnwrapped": "74.788", "lookAngle": "34.3", "farEndLat": 19.605, "incidenceAngle": null, "insarStackSize": "0", "centerLon": -155.3461, "product_file_id":

"ALPSR_22906_23577_0370_INTERFEROMETRY",

"processingLevel": "INTERFEROMETRY", "thumbnail": "none", $\quad$ "downloadUrl": "http://archive.daac.asf.alaska.edu:80/INTERFEROMETRY/A3 /ALPSR_22906_23577_0370.h5", "browse": "http://archive.daac.asf.alaska.edu:80/BROWSE/A3/ALPSR_22 906 23577 0370.jpg", "processingTypeName": "L1", "processingDate": "2014-03-19 22:48:35", "beamSwath": "7", "lookDirection": "R", "relativeOrbit": "291"\},..."ifgramCount": 3, "requestParameters": \{"platform": "ALOS", "frame": "370", "relativeOrbit": "291", "beamSwath": "7"\}\}

\subsection{API Keywords}

\begin{tabular}{|c|c|c|}
\hline QUERYFIELD & Description & $\begin{array}{c}\text { Example Input } \\
\text { Values }\end{array}$ \\
\hline absoluteOrbit & $\begin{array}{l}\text { List or range of } \\
\text { values for the orbit } \\
\text { number }\end{array}$ & $\begin{array}{l}\text { Integer value(s) of } \\
\text { the relative orbit }\end{array}$ \\
\hline $\begin{array}{l}\text { minBaselinePerp } \\
\text { maxBaselinePerp }\end{array}$ & $\begin{array}{l}\text { Baselines relative to } \\
\text { earliest scene in a } \\
\text { stack }\end{array}$ & $\begin{array}{l}\text { Integer value for } \\
\text { baseline }\end{array}$ \\
\hline beamMode & $\begin{array}{l}\text { Operating mode(s) } \\
\text { of the sensor } \\
\text { (comma separated } \\
\text { list) }\end{array}$ & $\begin{array}{l}\text { Fine, Standard, } \\
\text { Wide Swath }\end{array}$ \\
\hline beamSwath & $\begin{array}{l}\text { Swath name(s) of } \\
\text { the sensor (comma } \\
\text { separated list) }\end{array}$ & $\begin{array}{l}\text { ST1, ST2,...FN1, } \\
\text { FN2, .. }\end{array}$ \\
\hline collectionName & $\begin{array}{l}\text { List of collections } \\
\text { to include in search }\end{array}$ & $\begin{array}{l}\text { WInSAR, } \\
\text { EarthScope, CSA, } \\
\text { ASF }\end{array}$ \\
\hline $\begin{array}{l}\text { minDoppler } \\
\text { maxDoppler }\end{array}$ & $\begin{array}{l}\text { Values for the } \\
\text { Doppler centroid of } \\
\text { a scene }\end{array}$ & $\begin{array}{l}\text { Float value for } \\
\text { Doppler }\end{array}$ \\
\hline $\begin{array}{l}\text { minFaradayRotation } \\
\text { maxFaradayRotation }\end{array}$ & $\begin{array}{l}\text { Values for the } \\
\text { faraday rotation of a } \\
\text { scene }\end{array}$ & $\begin{array}{l}\text { Float value for the } \\
\text { faraday rotation }\end{array}$ \\
\hline flightDirection & $\begin{array}{l}\text { Specifies the } \\
\text { direction the } \\
\text { platform is traveling }\end{array}$ & $A$ or $D$ \\
\hline frame & $\begin{array}{l}\text { List or range of } \\
\text { values for the frame } \\
\text { number of the scene }\end{array}$ & $\begin{array}{l}\text { Integer value(s) for } \\
\text { the frame }\end{array}$ \\
\hline $\begin{array}{l}\text { minInsarStackSize } \\
\text { maxInsarStackSize }\end{array}$ & $\begin{array}{l}\text { Number of scenes } \\
\text { in InSAR } \\
\text { compatible stacks }\end{array}$ & $\begin{array}{l}\text { Integer value for } \\
\text { stack size }\end{array}$ \\
\hline intersectsWith & $\begin{array}{l}\text { Geographic area of } \\
\text { interest for the } \\
\text { search }\end{array}$ & $\begin{array}{l}\text { Well-known text } \\
\text { (WKT) format } \\
\text { POINT/LINE/POL } \\
\text { YGON }\end{array}$ \\
\hline lookDirection & $\begin{array}{l}\text { Specifies the } \\
\text { direction the sensor } \\
\text { is looking }\end{array}$ & $\mathrm{R}$ or $\mathrm{L}$ \\
\hline $\begin{array}{l}\text { masterStart } \\
\text { masterEnd }\end{array}$ & $\begin{array}{l}\text { Specify the start } \\
\text { date, end date, or } \\
\text { both of the master } \\
\text { granule of a } \\
\text { standarized sample } \\
\text { InSAR product. }\end{array}$ & $\begin{array}{l}\text { Example: } \\
\text { masterStart=2007- } \\
\text { 09-19 20:27:39 }\end{array}$ \\
\hline
\end{tabular}




\begin{tabular}{|c|c|c|}
\hline QUERYFIELD & Description & $\begin{array}{l}\text { Example Input } \\
\text { Values }\end{array}$ \\
\hline maxResults & $\begin{array}{l}\text { Maximum number } \\
\text { of scenes to return } \\
\text { from each archive }\end{array}$ & $\begin{array}{l}\begin{array}{l}\text { Integer value } \\
\text { (default } 1000 \text {, max } \\
10000 \text { ) }\end{array} \\
\end{array}$ \\
\hline minPercentCoherence & $\begin{array}{l}\text { Specify the } \\
\text { minimum } \\
\text { acceptable } \\
\text { percentage of } \\
\text { coherence on } \\
\text { standardized sample } \\
\text { InSAR products }\end{array}$ & $\begin{array}{l}\text { Integer value } 0 \text { - } \\
100\end{array}$ \\
\hline minPercentTroposphere & $\begin{array}{l}\text { Specify the } \\
\text { minimum } \\
\text { acceptable } \\
\text { percentage of } \\
\text { tropospheric } \\
\text { correction } \\
\text { performed on } \\
\text { standardized sample } \\
\text { InSAR products } \\
\text { example: }\end{array}$ & $\begin{array}{l}\text { Integer value } 0- \\
100\end{array}$ \\
\hline minPercentUnwrapped & $\begin{array}{l}\text { Specify the } \\
\text { minimum } \\
\text { acceptable } \\
\text { percentage of phase } \\
\text { unwrapping } \\
\text { performed on } \\
\text { standardized sample } \\
\text { InSAR products }\end{array}$ & $\begin{array}{l}\text { Integer value } 0- \\
100\end{array}$ \\
\hline output & $\begin{array}{l}\text { Desired return from } \\
\text { the result }\end{array}$ & $\begin{array}{l}\text { JSON (default), } \\
\text { KML, C }\end{array}$ \\
\hline platform & $\begin{array}{l}\text { Name of satellite or } \\
\text { airborne mission }\end{array}$ & $\begin{array}{l}\text { Long name of } \\
\text { platform (i.e. ERS- } \\
\text { 1, RADARSAT-1, } \\
\text { ENVISAT...) }\end{array}$ \\
\hline polarization & $\begin{array}{l}\text { Polarization } \\
\text { mode(s) of the } \\
\text { sensor (comma } \\
\text { separated list) }\end{array}$ & $\begin{array}{l}\mathrm{HH}, \mathrm{VV}, \mathrm{HV}, \mathrm{VH}, \\
\mathrm{HH}+\mathrm{HV}, \mathrm{VV}+\mathrm{VH},\end{array}$ \\
\hline processingLevel & $\begin{array}{l}\text { List of product } \\
\text { levels to includein } \\
\text { search }\end{array}$ & L0, SLC... \\
\hline relativeOrbit & $\begin{array}{l}\text { List or range of } \\
\text { values for the } \\
\text { "track" or "path" of } \\
\text { the scene }\end{array}$ & $\begin{array}{l}\text { Integer value(s) for } \\
\text { the relative orbit }\end{array}$ \\
\hline $\begin{array}{l}\text { slaveStart } \\
\text { slaveEnd }\end{array}$ & $\begin{array}{l}\text { Specify the start } \\
\text { date, end date, or } \\
\text { both of the slave } \\
\text { granule of a } \\
\text { standarized sample } \\
\text { InSAR product. }\end{array}$ & $\begin{array}{l}\text { Example: } \\
\text { slaveEnd=2011- } \\
\text { 02-15 00:00:00 }\end{array}$ \\
\hline $\begin{array}{l}\text { start } \\
\text { end }\end{array}$ & $\begin{array}{l}\text { Date of data } \\
\text { acquisition; enter a } \\
\text { start date, end date, } \\
\text { or both to form a } \\
\text { valid range }\end{array}$ & $\begin{array}{l}\text { Example: } \\
\text { start=2010-10- } \\
\text { 30T11:59:59UTC } \\
\text { \&end=1991-10- } \\
\text { 01T00:00:00UTC }\end{array}$ \\
\hline
\end{tabular}

Table 12. SSARA API Keywords.

\subsection{Resources}

1. SSARA SAR Service:

http://www.unavco.org/ws/brokered/ssara/sar/search

2. SSARA InSAR Pair Service:

http://www.unavco.org/ws/brokered/ssara/insar/selectpairs

3. SSARA InSAR Product Service:

http://www.unavco.org/ws/brokered/ssara/insar/search

4. DEM Service: http://ot-data1.sdsc.edu:9090/otr/getdem

5. Tropospheric Service: http://oscar.jpl.nasa.gov
6. SSARA GitHub Page:

https://github.com/bakerunavco/SSARA

7. SSARA GitHub Wiki:

https://github.com/bakerunavco/SSARA/wiki

\section{REFERENCES}

\section{ACKNOWLEDGEMENTS}

This work was made possible by the 2011 NASA ROSES ACCESS Program (NASA Proposal Number 11-ACCESS110035).

InSAR Product, SSARA software, testing, and documentation contributions provided by Rudi Gens ${ }^{\mathrm{b}}$, Tim Stern ${ }^{\mathrm{b}}$, Kirk Hogenson ${ }^{b}$, and Greg Moore ${ }^{c}$.

\section{REFERENCES}

Crosby, C., Arrowsmith, J., Nandigam, V., Baru, C., 2011. A Geoinformatics Approach To Online Access And Processing Of LIDAR Topography Data. In: Geoinformatics, R. Keller and C. Baru, eds., Cambridge University Press, London.

Hanssen, R., 2001. Radar Interferometry: Data Interpretation and Error Analysis. Kluwer Academic Publishers.

Krishnan, S., Crosby, C., Nandigam, V., Phan, P., Cowart, C., Baru, C., and Arrowsmith, J., 2011. OpenTopography: a services oriented architecture for community access to LIDAR topography. In: Proceedings of the 2nd International Conference on Computing for Geospatial Research \& Applications (COM.Geo '11), AMC, pp. 8. DOI $=10.1145 / 1999320.1999327$

Pepe, A. and Lanari, R., 2006. On the extension of the minimum cost flow algorithm for phase unwrapping of multitemporal differential SAR interferograms, IEEE Trans. Geosci. Remote Sens., 44, 9, pp. 2374-2383.

Youn, C.,Nandigam, V., Phan, M., Tarboton, D., WilkinsDiehr, N., Baru, C., Crosby, C., Padmanabhan, A., and Wang, S., 2014. Leveraging XSEDE HPC resources to address computational challenges with high-resolution topography data. In Proceedings of the 2014 Annual Conference on Extreme Science and Engineering Discovery Environment (XSEDE '14). ACM, New York, NY, USA, , Article 59, pp 2. DOI $=10.1145 / 2616498.2616564$

Li, Z., Fielding, E. J., Cross, P., and Muller, J.-P., 2006, InSAR atmospheric correction GPS Topography- dependent Turbulence Model (GTTM): Journal of Geophysical Research, v. 111, pp. B02404. DOI:02410.01029/02005JB003711.

Li, Z., Muller, J., Cross, P., and Fielding, E., 2005, InSAR atmospheric correction: GPS, MODIS and InSAR integration: Journal of Geophysical Research, v. 110, p. B03410. DOI:03410.01029/02004JB003446.

von Allmen, P., Fielding, E., Fishbein, E., Li, Z., Xing, Z., Pan, L., 2011. OSCAR: Online Services for Correcting Atmosphere in Radar. https://earth.esa.int/documents/10174/1566700/Allmen_FRING E2011.pdf 